\title{
“PINOCCHIO": A Rare Case Report
}

${ }^{1}$ BP Belaldavar, ${ }^{2}$ Prashant H Patil, ${ }^{3}$ Prakash R Malur, ${ }^{4}$ Shailashree L Samaga, ${ }^{5}$ Harshalatha S Mahajan

${ }^{6}$ Ashwath Kasliwal, ${ }^{7}$ Suhasini Hanumaiah

\section{ABSTRACT}

Aim: To present a rare case of nasal dermoid cyst and its management in a 14-year-old boy.

Background: Congenital midline nasal masses are rare, with an incidence estimated to be 1 in 20,000 to 1 in 40,000. Among these, nasal dermoid cyst is commonest. They arise from the lines of embryonic fusion. Various theories have been postulated in the development of nasal dermoid cyst, and prenasal theory by Pratt is the most accepted one.

Case report: A 14-year-old boy presented with a gradually progressive midline swelling since birth, which was solitary, soft, nontransilluminant, present over the lower dorsum and tip region. Based on the history, clinical examination, and serial relevant investigations, diagnosis of a nasal dermoid cyst was made. Surgical strategy planned was dermoid cyst extirpation via an open rhinoplasty approach and augmentation of the defect with an autologous conchal cartilage graft.

Conclusion: Nasal dermoid cyst is a rare congenital anomaly. Presence of intracranial extensions, sinus formation, and other differential diagnosis should be ruled out preoperatively. It demands early surgical intervention in order to avoid further distortion of the nose or atrophy of the bony or cartilaginous component as a result of pressure secondary to the growth of cyst and infection.

\section{Clinical significance:}

- This midline congenital cyst is cosmetically unacceptable. Hence, early surgical removal is mandatory.

- Open rhinoplasty is the preferred surgery as it gives good esthetic results. It gives a scope for augmentation of the defect following the extirpation of the cyst.

- Autologous conchal cartilage is the preferred graft as it provides sufficient quantity and esthetically pleasing contour.

Keywords: Augmentation rhinoplasty, Autologous conchal cartilage, Nasal dermoid cyst, Open rhinoplasty.

How to cite this article: Belaldavar BP, Patil PH, Malur PR, Samaga SL, Mahajan HS, Kasliwal A, Hanumaiah S. "PINOCCHIO": A Rare Case Report. Clin Rhinol An Int J 2017; 10(3):142-146.

\footnotetext{
${ }^{1,2}$ Professor, ${ }^{3} \mathrm{Head},{ }^{4-7}$ Resident

1,2,4-7 Department of ENT and Head and Neck Surgery, Jawaharlal Nehru Medical College, KLE University, Belagavi, Karnataka India

${ }^{3}$ Department of Pathology, KLE Hospital, Belagavi, Karnataka India

Corresponding Author: BP Belaldavar, Professor, Department of ENT and Head and Neck Surgery, Jawaharlal Nehru Medical College, KLE University, Belagavi, Karnataka, India, Phone: +919845274729, e-mail: puttawwa@rediffmail.com
}

\section{Source of support: Nil}

Conflict of interest: None

\section{INTRODUCTION}

Nasal dermoid cyst is a rare developmental anomaly that often arises in lines of embryonic fusion. It is derived from ectoderm and mesoderm and can contain all structures of normal skin. Nasal dermoid cysts comprise 1 to $3 \%$ of all dermoid cysts and 4 to $12 \%$ of head and neck dermoids. ${ }^{1}$ They are most common congenital midline nasal masses, which may arise from glabella to columella, nasoglabellar mass being the most common site of presentation. ${ }^{2-4}$ They may appear as a cystic mass with a sinus opening or may present with nasal obstruction or broadening of nasal root or bridge. It usually terminates in a subcutaneous tract or with intracranial connection. Through the subcutaneous tract it may secrete sebaceous material or pus. It may get infected intermittently and form an abscess, thereby resulting in osteomyelitis. Intracranial connections can result in meningitis and cerebral abscess, which are seen in 4 to $45 \%$ of cases. Hence, radiological evaluation is mandatory prior to biopsy. Treatment option is surgical excision via open rhinoplasty, which is the preferred approach. ${ }^{5}$

\section{CASE REPORT}

A 14-year-old boy presented with swelling over tip of the nose since birth. Initially, it was size of a jowar seed that has gradually progressed to size of a gooseberry over 14 years, with no associated pain, discharge, or any change in color. There was no history of fever, headache, and vomiting.

On inspection, a solitary, sessile, spherical, midline swelling over tip of the nose, measuring about $3 \times 3 \mathrm{~cm}$, smooth, nonerythematous was seen. On palpation, the swelling was soft, no tender, well-demarcated, fluctuant, compressible, nonreducible, nontransilluminant, and slip sign was negative (Figs 1 and 2).

\section{INVESTIGATIONS}

Routine blood investigations: Normal

$X$-ray nose lateral view: Soft tissue swelling noted at the lower dorsum over the tip of nose. Nasal bones are normal (Fig. 3). 


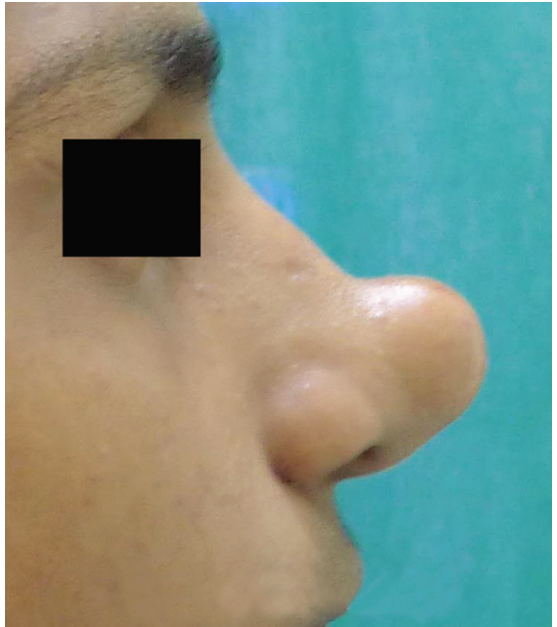

Fig. 1: Swelling over the tip of the nose

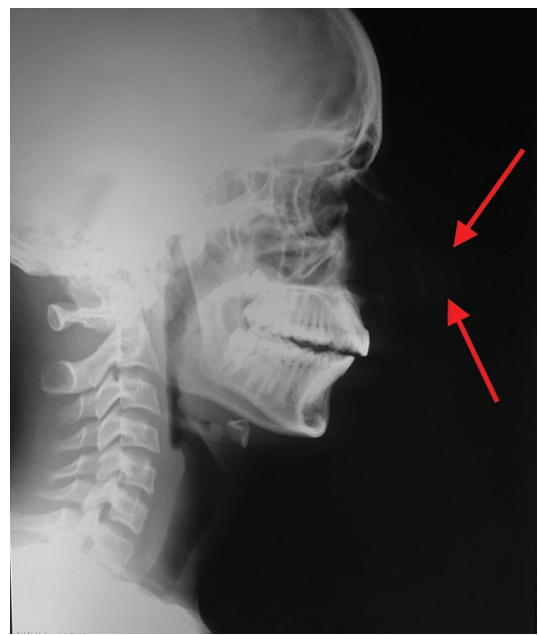

Fig. 3: X-ray nose lateral view: soft tissue swelling noted at the lower dorsum over the tip of nose

Ultrasonography nose: Well-defined cystic collection with thick internal contents at tip of the nose along midline, measuring $2.4 \times 2.2 \times 1.6 \mathrm{~cm}$. On color Doppler, collection shows no vascularity, suggestive of dermoid cyst.

Fine needle aspiration cytology: Papanicolaou and MayGrunwald-Giemsa stained smears: Plenty of anucleate squames in the background of keratin debris suggestive of dermoid cyst. Aspirate was sent for culture: No organisms grown.

Computed tomography (CT) scan plain and contrast: Well-defined hypodense soft tissue lesion showing fatty attenuation at the tip of the nose along the midline (Fig. 4).

Treatment: Excision of the dermoid cyst was through an open rhinoplasty approach with augmentation rhinoplasty using conchal cartilage (Fig. 5).

\section{HISTOPATHOLOGY REPORT}

Gross: Oval, cystic, soft tissue piece, measuring $2 \times 2 \times 1$ $\mathrm{cm}$. Cut section contained putty-like material.

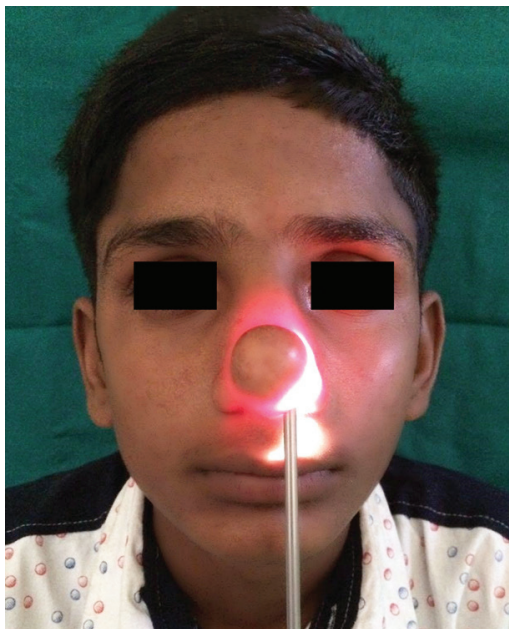

Fig. 2: Negative transillumination test

Microscopy: The cyst is lined by stratified squamous epithelial cells, keratin debris, and adjacent layer of cartilage (Fig. 6).

\section{DISCUSSION}

External nasal masses are rare, and congenital masses are even rarer of the all. Further, among the congenital masses, congenital midline nasal masses being the rarest, occur one in every 20,000 to 40,000 live births. ${ }^{1,6,7}$ The term "nasal dermal cyst" was coined by Session. ${ }^{8}$ It is formed by three layers. They are ectoderm, mesoderm, and the deeper layer of cartilaginous capsule. The first description of a nasal dermoid cyst was given by Cruvelier. He described nasal dermoid cyst in a child which presented with a sinus-containing hair that opened on the dorsum of the nose.

Various theories have been postulated in the development of dermoid cyst, such as superficial sequestration theory by Bland-Sutton, ${ }^{9}$ trilaminar theory as proposed by Littlewood. Prenasal theory by Pratt and cranial theory by Bradley are the most accepted ones. ${ }^{2}$ Development of skull base and nose is a complex process. It involves formation of mesenchymal structures which are formed as a result of fusion and ossification of several centers. There are recognized spaces in between these centers which are responsible for the development of congenital midline nasal masses. These include fonticulus nasofrontalis, the prenasal space, and the foramen cecum that eventually get closed by fusion and ossification. ${ }^{3,10}$ Due to failure of the fusion of frontal and nasal bones, dura extends through the foramen cecum and through the prenasal space to come into contact with the epidermis of the skin, resulting in trapping of ectodermal elements. Usually, the dural projection loses its cutaneous connection and retracts through the foramen cecum, followed by closure of the latter. If this process of obliteration is incomplete, 

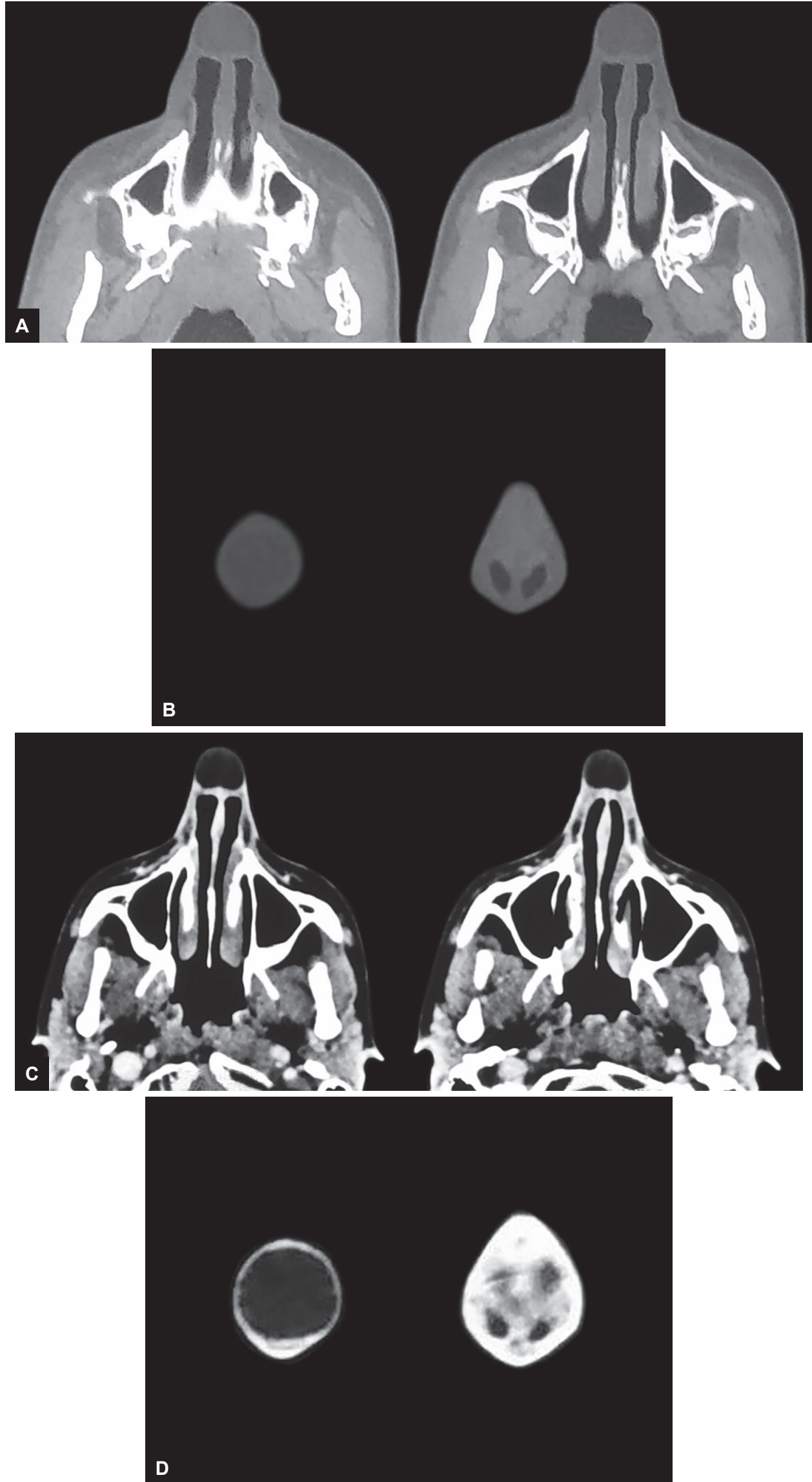

Figs 4A to D: (A) CT scan plain showing axial and coronal view well-defined hypodense lesion over tip of the nose; (B) CT scan plain showing axial and coronal view well-defined hypodense lesion over tip of the nose; (C) CT scan with contrast: well-defined hypodense soft tissue lesion showing fatty attenuation in the tip of the nose along the midline; and (D) CT scan with contrast: well-defined hypodense soft tissue lesion showing fatty attenuation in the tip of the nose along the midline 

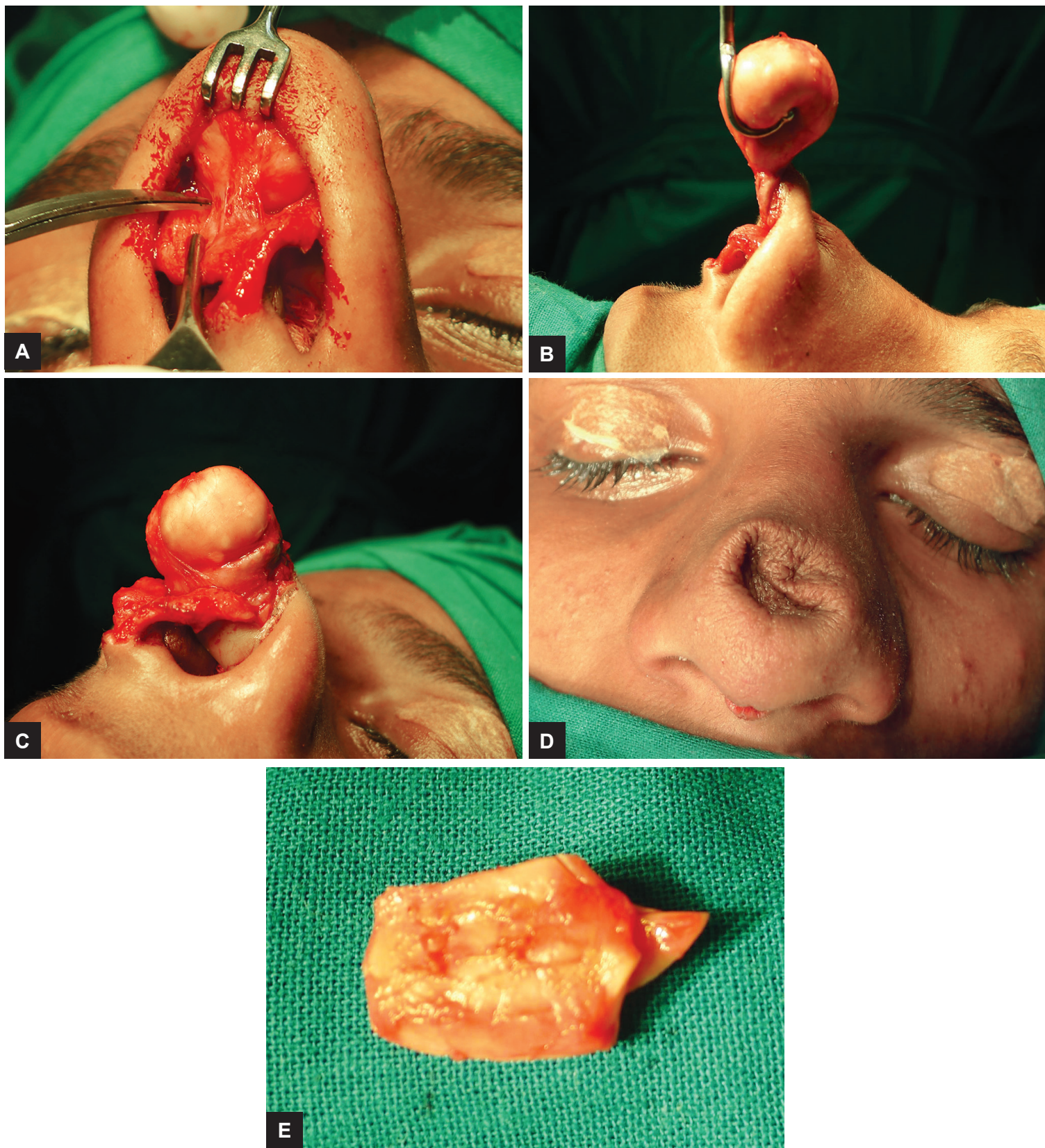

Figs 5A to E: Intraoperative images: (A) exposure of ligament of Pitanguy and the cyst; (B) dermoid cyst dissected and separated from its attachment to lower alar cartilage. Skin pinched in so as to pop out the cyst; (C) cyst separated from all the sides in toto except from its superior attachment. Later even superior attachment dissected; (D) defect in the supratip area following cyst removal; and $(E)$ conchal cartilage harvested and sculptured to an appropriate shape

dermal connections in the form of a sinus tract or a cyst may be found at any level of the pathway. The insidious growth of nasal dermoids, the risk of meningitis, and the cosmetic deformity are indications for surgical extirpation. Differential diagnosis that has to be ruled out are gliomas, encephaloceles, and epidermoid cysts. ${ }^{1,6}$ Imaging modalities for diagnosis are plain radiographs, CT scan, and magnetic resonance imaging; plain and with contrast that helps to access the lesion and determine possible intracranial extensions if present. ${ }^{2,4}$

In this case, a 14-year-old boy presented to us mimicking and reminding us of the well-known cartoon character "PINOCCHIO." This swelling had the charac-

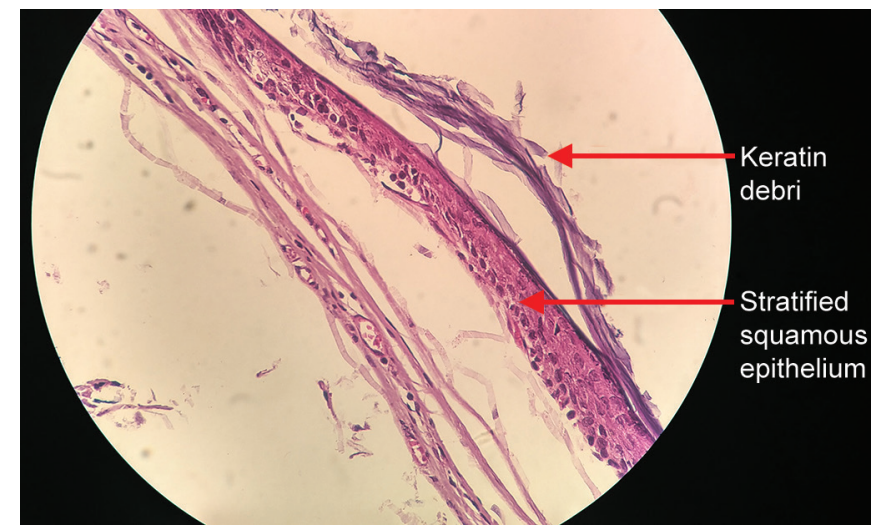

Fig. 6: Histopathology: stratified squamous epithelial cells, keratin debris 


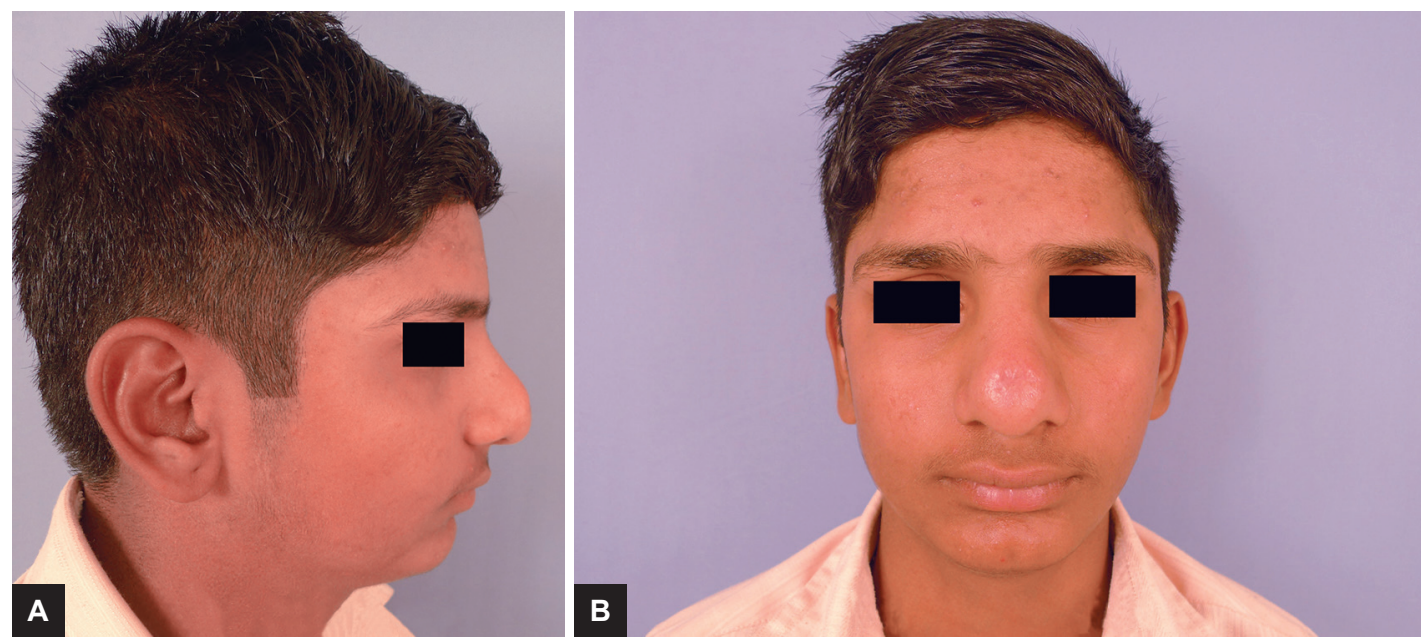

Figs 7A and B: (A) The location and position of the graft beneath the skin over the lower alar cartilages and in between upper alar cartilages; $(B)$ postoperative images: sixth month postoperative

teristic findings of a dermoid cyst with a rare presentation over lower dorsum of the nose and featureless tip. Diagnosis was confirmed with serial relevant investigations. Excision of the dermoid cyst via open rhinoplasty was performed. Supratip lower dorsum defect (saddling) was corrected with morselized and crushed stack of conchal cartilage graft (Fig. 5). Postoperative day 10 dressing and sutures were removed. Thereafter, patient was called for regular follow-up and was uneventful (Fig. 7: postoperative images).

\section{CONCLUSION}

Swelling over the dorsum of nose as seen in this case is cosmetically unacceptable. Open rhinoplasty provides better cosmetic results, wide exposure, and scope for reconstruction in order to reinforce the nasal dorsal skin, better healing, and less chances of gaping wound; bimanual surgical dissection can be accomplished more efficiently and a very good technique for demonstration and training of the residents. Moreover, external rhinoplasty gives a very good access to the tip of the nose. ${ }^{3,11,12}$

Most repairs of nasal defects, after nasal dermoid cyst excision, are done by using an open rhinoplasty approach with autologous septal and costal cartilages as graft materials for reconstruction. Autologous cartilage is the material of choice for dorsal augmentation. ${ }^{5}$ Reconstructing the defect using only septal cartilage is insufficient for dorsal augmentation, whereas costal cartilage has been associated with donor site morbidity. Moreover, Asians have a relatively smaller septal cartilage and in younger patients the amount of cartilage is quite small, thereby requiring the preservation of large L-strut. Therefore, in this case, autologous conchal cartilage was used as there was obvious defect at supra tip area causing the saddling, and the amount of harvested conchal cartilage was sufficient enough to accommodate the same and give stability and good cosmetic contour.

\section{REFERENCES}

1. Tatar EC, Selcuk ÖT, Saylam G, Özdek A, Korkmaz H. The management of rare nasal mass-nasal dermoid sinus cysts: open rhinoplasty. Rare Tumors 2009 Dec;1(2):e40.

2. Rahbar R, Shah P, Mulliken JB, Robson CD, Perez-Atyade AR, Proctor MR, Kenna MA, Scott MR, McGill TJ, Healy GB. The presentation and management of nasal dermoid. Arch Otolaryngol Head Neck Surg 2003 Apr;129(4):464-471.

3. Al-Reefy H, Hopkins $\mathrm{C}$, Jonas N. The open access atlas of otolaryngology, head and neck operative surgery. Surgery for Nasal Dermoids. www.entdev.uct.ac.za

4. Guruprasad Y, Chauhan DS. Midline nasal dermoid cyst with Tessiers 0 cleft. J Nat Sci Biol Med 2014 Jul;5(2):479-482.

5. Kim JH, Wang JH, Jang YJ. Excision of a nasal dermoid sinus cyst via open rhinoplasty approach and primary reconstruction using tutoplast-processed fascia lata. Clin Exp Otorhinolaryngol 2010 Mar;3(1): 48-51.

6. Wang IJ, Lin SL, Tsou KI, Hsu MC, Chiu WT, Tsai ST, Lee LM, Lin TJ. Congenital nasal midline mass: case series and review of literature. Turk J Paediatr 2010 Sep-Oct;52(5):520-524.

7. Yildiz H, Cil Y, Simsek HA, Erginay T. Recurrent adult nasal dermoid cyst. J Cutan Aesthet Surg 2011 May-Aug;4(2): 151-152.

8. Sessions RB. Nasal dermal sinuses: new concepts and explanations. Laryngoscope 1982 Aug;92(8 Pt 2 Suppl 29):1-28.

9. Bland SJ. A note on dermoid cysts of the scalp and mid-line of the back. Ann Surg 1889 Jul-Dec;10:81-86.

10. Pratt LW. Midline cysts of nasal dorsum: embryologic origin and treatment. Laryngoscope 1965 Jun;75:968-980.

11. Gupta M, Chaudhary N. External rhinoplasty for dorsum swellings. Indian J Otolaryngol Head Neck Surg 2007 JanMar;59(1):60-62.

12. Sood, VP. Corrective rhinoplasty. 3rd ed. New Delhi: CBS Publishers \& Distributors Pvt. Ltd.; 2013. pp. 134-139. 